\title{
Sunflower Seed and Acne Vulgaris
}

\author{
Alireza Mohebbipour ${ }^{1}$; Homayoun Sadeghi-Bazargani ${ }^{2,{ }^{*}} ;$ Mona Mansouri $^{1}$ \\ ${ }^{1}$ Department of Dermatology, Faculty of Medicine, Ardabil Branch, Islamic Azad University, Ardabil, IR Iran \\ ${ }^{2}$ Road Traffic Injury Research Center, Department of Statistics and Epidemiology, Tabriz University of Medical Sciences, Tabriz, IR Iran \\ ${ }^{*}$ Corresponding Author: Homayoun Sadeghi-Bazargani, Road Traffic Injury Research Center, Department of Statistics and Epidemiology, Tabriz University of Medical Sciences, Tabriz, \\ IR Iran. Tel:+98-9144027218, E-mail: homayoun.sadeghi@gmail.com
}

Received: January 2, 2014; Revised: October 31, 2014; Accepted: December 14, 2014

\begin{abstract}
Background: Regardless of the overall association between diet and acne which cannot be easily ignored, there might be an association between specific nutrients and acne development or improvement.

Objectives: The aim of this study was to assess the effect of dietary intake of sunflower seeds on acne severity and the pattern of acne lesions.

Patients and Methods: In a randomized controlled trial, 50 patients aged 15 - 30 years old with acne vulgaris were enrolled through consecutive convenient sampling, in a dermatology clinic in Ardabil, Iran. They were randomly allocated into two trial arms. Those in the control group were asked to stop eating sunflower seeds if they did before. In the intervention group, they consumed $25 \mathrm{~g}$ sunflowercontaining food daily for seven days. The primary outcome of interest was 10\% increase/decrease in the baseline acne severity index (ASI), sustained to the end of the follow-up period on day 14.

Results: The mean ASI did not change significantly through the study period in the control group, but it increased in the sunflower group from 62 at the baseline to 86.8 after two weeks $(\mathrm{P}<0.001)$. The ASI mean change was 24.8 in the sunflower group compared to 4.9 in the control group $(\mathrm{P}<0.001)$. The global acne grading score (GAGS) did not significantly change in any of the groups and the difference in the change of GAGS was not significant between the groups (2.4 in the sunflower group versus 1.6 in the control group). Twenty two subjects (88\%) in the sunflower group versus 9 (36\%) in the control group had at least 10\% increment in ASI throughout the follow-up period $(\mathrm{P}<0.001)$. The relative risk of developing the primary outcome in taking the sunflower seed intervention was 2.4 (95\% CI: 1.4 - 4.2). The observed risk difference was 0.52 (95\% CI: 0.29 - 0.75).

Conclusions:Sunflower seed intake appears to aggravate acne vulgaris; however, further evidence is needed to ban sunflower seed intake in patients with acne. Considering the observed potential negative effect in this trial, future randomized clinical trials may base their design on randomly assigning the exposed patients to give up use of sunflower seed intake.
\end{abstract}

Keywords: Acne Vulgaris; Diet; Sunflower; Dietary Fats; Feeding Pattern

\section{Background}

The association between diet and acne has been highly controversial in dermatological practice and research history. Regardless of the overall relationship between diet and acne which cannot be easily ignored, research may clarify the possible association between specific nutrients and acne development or improvement (1-3). Based on evidence regarding the anti-inflammatory effects of free fatty acids like linoleic and lauric acid in sebum to inhibit Propionibacterium acnes, herbs containing linoleic acid are thought to be applicable in acne therapy (4). Therefore, sunflower and pumpkin seed oils as well as flax or linseed oil have been used as a preparation for dermatological treatments including acne (5). Regardless of the question that how strong is the theory on the efficacy of topical sunflower seed oil in treating acne, it is important to find out if eating sunflower seeds that is a common nutritional habit in some areas can affect the development or the severity of acne vulgaris. This is while reliable evidence to be introduced by clinical trials is not available to show the efficacy of oral sunflower seed in improving or aggravating acne.

\section{Objectives}

The aim of this pilot study was to assess the effect of dietary intake of sunflower seeds on severity of acne and the pattern of acne lesions.

\section{Patients and Methods}

In a randomized controlled trial in 2009, 50 patients aged 15 - 30 years old with acne vulgaris were enrolled. The patients were enrolled through consecutive convenient sampling method from an outpatient dermatology clinic in Ardabil province, northwest of Iran. They were randomly allocated into two trial arms as intervention and control group arms, using block randomization. The randomization sequence was generated using Microsoft Excel software package (6). It was concealed using alloca- 
tion labels given by one of the reception staff according to the sequence and the patients' order in the clinic registration list. It was implemented by the physician according to the label provided by the reception desk.

Those in the control group were asked to stop eating sunflower seeds if they did before. The intervention group consumed $25 \mathrm{~g}$ sunflower-containing food daily for seven days. This is the current form of packaging sunflower seeds which people in Iran are used to buying and consuming in each single occasion. The consumption period was selected not to be longer than one week due to safety considerations, as this was the first interventional research experience in this field. Although consuming sunflower seeds is a very enjoyable way of participating in a clinical trial, to ensure higher compliance, the dosage was also chosen based on the current consumption habits of Iranian people. The subjects were also asked whether they had used it per-protocol. All the patients were assessed for their acne status at baseline, after seven days and finally after 14 days. Due to limited available data in the literature, the only logic for choosing this evaluation time was the personal practice experience of the first author who had noticed some patients complaining of aggravating acne lesions 10-14 days after consuming the seeds.

The evaluations were conducted by an experienced dermatologist. Blinding was not possible in this study and randomization was performed after the informed consents were obtained. Clinical evaluations were performed according to the available recommended clinical evaluation tools recommended internationally and used also in previous Iranian studies. To assess the acne severity, a precisely defined severity tool called acne severity index (ASI) was applied. The formula used to calculate ASI was $2 \times$ pustules $+1 \times$ papules $+0.25 \times$ comedones. Moreover, global acne grading score (GAGS) was also used as a previously used acne grading scale (7-9).

To test an equality hypothesis, the primary outcome of interest was occurrence of $10 \%$ increase/decrease in baseline ASI, sustained to the end of the follow-up period. Other outcomes assessed in this study were global acne GAGS, number of comedones, papules, nodules, and pustules.

The exclusion criteria were; acne conglobata, cystic nodular acne, Gram-negative folliculitis, fulminans, acne therapy in the recent three months, and using drugs that cause acne.

A primary power analysis was conducted to distinguish $30 \%$ difference of $5 \%$ presumed background aggravation rate, accepting 5\% type 1 error and 20\% type 2 errors. This yielded a sample size of 66 . Seventy-two patients were assessed for eligibility and 22 were excluded due to the exclusion criteria. Due to ethical considerations and unavailability of reliable information in power analysis, the consultation board recommended the sample size to be as low as possible and approved the study as a pilot study; thus, the randomization was conducted on 50 patients (Figure 1).

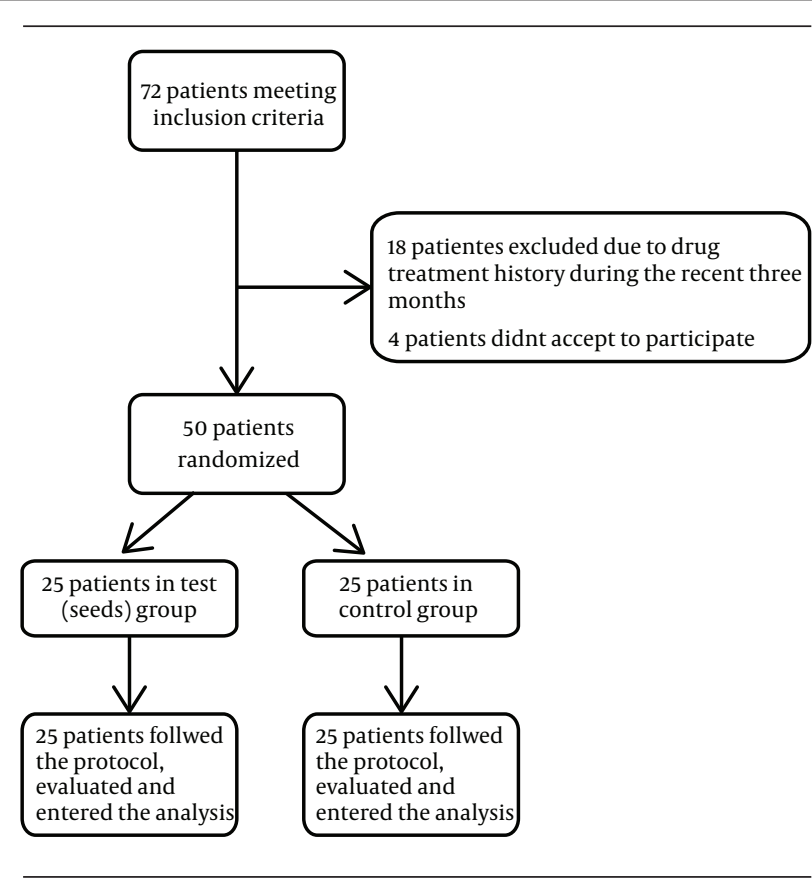

Figure 1. Consort Flowchart of the Trial

Data were entered into the computer and analyzed using Stata 11 statistical software package. Both descriptive and analytical methods were used. The difference in the mean ASI and the mean GAGS were initially tested using paired and independent t-tests. The groups were compared regarding the primary outcome of the study using initial chi-square and Fisher's exact tests, followed by the calculation of relative and absolute risk differences of the groups along with their 95\% confidence intervals (CIs). The traditional Poisson regression for counting data assumes statistical independence of observations, which is not usually held for multiple measurements over time. However, Poisson regression methods for longitudinal data have been introduced to overcome such limitation and it was applied in this study to compare the trends of acne lesion counts between the groups (10). Considering that we could not assume a directional hypothesis, all the tests were performed as two-tailed. A P value $<0.05$ was considered as the level of statistical significance. The study protocol was approved in 2009 by the responsible committee for research and ethics in Islamic Azad University (Ardabil) to the responsibility of the first author as the main researcher. The ethical code for the regional committee of ethics was ARUMS.Rec.93.54. Verbal informed consents were obtained from all the participants, letting them know about possible benefits and risks of the investigation and they were assured of the confidentiality of information they provided.

\section{Results}

Of all the 50 patients, 36 (72\%) were female. The mean age of the participants was $23.5 \pm 4.6$ years without any statistically significant difference between the groups. 
Seventy four percent of the participants had acne history in their family. A history of previous treatment was found in $62 \%$. A history of coincident hirsutism was observed in one-fifth of the patients and $20 \%$ also complained of oily skin. Mean count and count standard deviation of different acne lesions compared over the follow-up time and also between the two groups are descriptively presented in Table 1. Count trends of different acne lesions were compared between the trial groups using Poisson regression for longitudinal data, which did not find statistically different trends in spite of a descriptively larger slope observed in the sunflower group.

As shown in Figure 2, the mean ASI did not significantly change over time in the control group, but it increased in the sunflower group from 62 in baseline to 86.8 after two weeks ( $\mathrm{P}<0.001)$. The ASI mean change was 24.8 in the sunflower group compared to 4.9 in the control group. The change difference between the groups was statistically significant $(\mathrm{P}<0.001)$.

As shown in Figure 3, GAGS score did not significantly change in any of the groups and the difference in change of GAGS score was not also different between the groups (2.4 in the sunflower group versus 1.6 in the control group).

Twenty two patients (88\%) in the sunflower group versus $9(36 \%)$ in the control group developed at least $10 \%$ increment in ASI throughout the follow-up period $(\mathrm{P}<$ 0.001). The relative risk of developing the primary outcome in taking sunflower seed intervention was 2.4 (95\% CI: 1.4 - 4.2). The observed risk difference was 0.52 (95\% CI: $0.29-0.75)$.

Table 1. Mean Count and Standard Deviation of Different Acne Lesions Compared Over the Follow-up Time Between the Two Groups ${ }^{\text {a }}$

\begin{tabular}{|c|c|c|}
\hline Lesion Type/Time & Sunflower Group & Control Group \\
\hline \multicolumn{3}{|l|}{ Comedone count } \\
\hline Baseline & $5.3 \pm 2$ & $5 \pm 1.6$ \\
\hline $7 \mathrm{~d}$ & $5.8 \pm 2.1$ & $5.2 \pm 1.6$ \\
\hline $14 \mathrm{~d}$ & $6.2 \pm 2.27$ & $5.3 \pm 1.84$ \\
\hline \multicolumn{3}{|l|}{ Papule count } \\
\hline Baseline & $1.3 \pm 0.83$ & $1.3 \pm 0.61$ \\
\hline $7 \mathrm{~d}$ & $1.7 \pm 0.91$ & $1.5 \pm 1$ \\
\hline $14 \mathrm{~d}$ & $2.2 \pm 0.97$ & $1.6 \pm 0.67$ \\
\hline \multicolumn{3}{|l|}{ Pustule count } \\
\hline Baseline & $1 \pm 0.6$ & $0.9 \pm 0.65$ \\
\hline $7 \mathrm{~d}$ & $1.4 \pm 0.72$ & $1.1 \pm 0.61$ \\
\hline $14 \mathrm{~d}$ & $1.6 \pm 0.84$ & $1.1 \pm 0.75$ \\
\hline \multicolumn{3}{|l|}{ Nodule count } \\
\hline Baseline & $1 \pm 0.56$ & $1.3 \pm 0.66$ \\
\hline $7 \mathrm{~d}$ & $1.1 \pm 0.59$ & $1.2 \pm 0.76$ \\
\hline $14 \mathrm{~d}$ & $1.5 \pm 0.76$ & $1.3 \pm 0.85$ \\
\hline
\end{tabular}

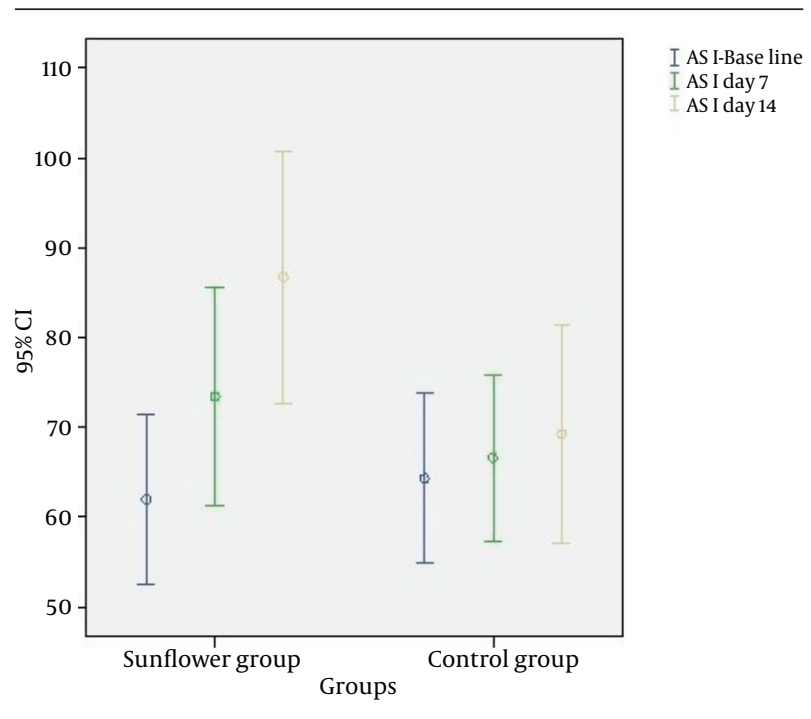

Figure 2. Error Bar Graph of Mean ASI Score in Three Occasions Compared Between Trial Groups

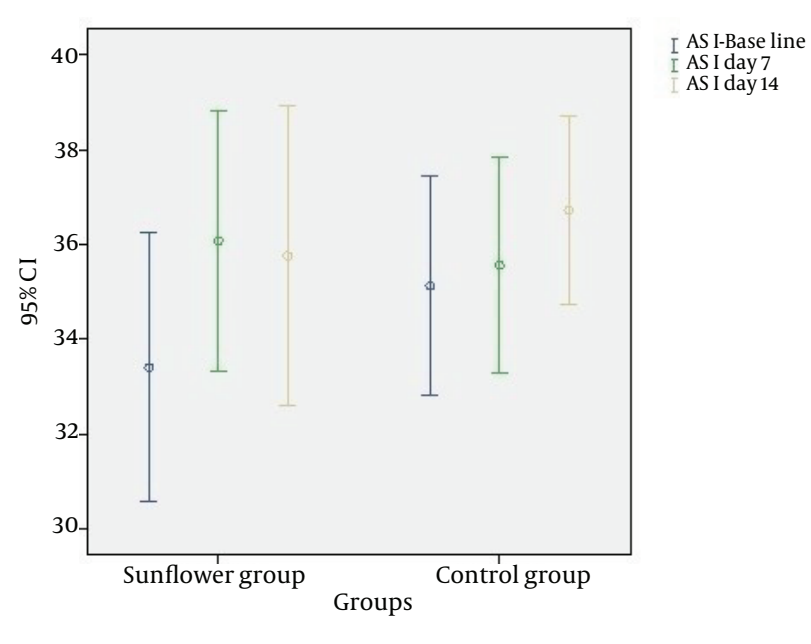

Figure 3. Error Bar Graph of Mean GAGS Score in Three Occasions Compared Between Trial Groups

\section{Discussion}

Our findings showed an aggravating effect for the intake of sunflower seeds on acne vulgaris. This study detected such an effect to be prominent on the ASI index. The results of the present study can be considered internally consistent, because of the coherence of the results in three aspects. First, the before-after aggravation was only observed in the investigational arm and a significant effect was not observed in the control group. Second, the mean change in ASI index was statistically different between the groups with a relatively high mean difference. Finally, taking into account the clinical significance difference of $10 \%$ as a basis for dichotomizing the effect measure led to a relative risk equal to 2.4 (95\% CI: 1.4 - 4.2). Although very few studies have specifically addressed 
the association of sunflower seed intake with acne, most of the previous researches have been performed on dietary groups, such as foods with high percentages of carbohydrate or oil in them. Most of the studies on diet and acne have been observational, mostly cross-sectional, case-control, and a few cohort studies (11). Clinical trial studies constituted a small share in the related literature. Very few controlled clinical trial studies have addressed the effects of diet on acne (12-14). Chocolate and milk have gained more attention in diet research, the latter being the most frequently cited acne-causing food (11).

One plausible theory to explain the possible association of high carbohydrate or oily food intake with acne may be the development of insulin resistance and a resulting cascade of increased androgens, increased insulin-like growth factor (IGF)-1 and altered retinoid signaling pathways $(2,15,16)$. As another aspect of plausibility, based on the known association of acne severity with facial sebum secretion, it has been hypothesized that foods high in fat or carbohydrates may exacerbate acne by production of more comedogenic sebum by increasing blood lipid levels or by producing sebum that is less fluid, which may in turn obstruct pilosebaceous follicles (2). However, contrary to animal studies consistently showing increases in insulin resistance on high-fat diets, this is controversial in humans studies (17). The general consensus among the experts is that the available evidence is insufficient to prove such association (2).

The specific role of sunflower seed in etiology or treatment of acne vulgaris has been stated in several studies. Most of these studies have considered a therapeutic effect for its topical products. The theory discussed in these studies has been mainly based on fatty acid gradients of sunflower seed and relating it to the anti-inflammatory effects of free fatty acids in sebum which were found to inhibit $P$. acnes $(4,5)$. Randomized treatment-specific trials have not been the base of conclusions made on efficacy of topical form of products with sunflower seeds. Few available studies were based of using multigradient topical products without strong methodological requirements to conclude on isolated efficacy of sunflower seeds or even the efficacy of combined products. The most recent review supporting the use of topical products in this regard states that the anti-inflammatory effects of free fatty acids in sebum, particularly linoleic and lauric acids, have been found to inhibit P. acnes. The authors then concluded that plants containing linoleic acid may be applicable in acne lesion reduction. Referring to the two previous articles, they considered naming of some plants like sunflower and pumpkin seed oils as well as flax or linseed oil, which have a high fatty acid content, mainly linoleic and linolenic acids, which were incorporated into a preparation for dermatological treatments including acne $(4,5,18)$. However, the same review stated that evaluation of these vegetable oils in acne treatment should be performed. Even if we consider positive effect for topical sunflower seed on acne, this cannot discard a reverse ef- fect of oral consumption of sunflower seeds. No doubt, much research is needed to confirm or clarify these observations, including basic science research, necessary for developing a plausible theory.

Regarding the sunflower seed intake, a study in Turkey found eating sunflower seed as a possible aggravating factor for acne vulgaris. However, the study was mainly comparative rather than controlled interventional. Based on a wide literature review in the present study, it seems that our study was possibly one of the first randomized clinical trials to assess the effects of sunflower seed on acne vulgaris.

In this study, we observed some improvements in the control group. However, it was just a descriptive change and was not statistically significant, indicating a high chance of random error if trying to extrapolate sample observations. Nevertheless, an explanation for this finding can be such that when someone finds his/her acne status to be bothersome enough to seek medical consultation, this may also convince the patient to make some nutritional or health behavioral changes, which in turn may affect the acne severity. Furthermore, we would like to stress that our methodology addressed an aggravating effect of sunflower seeds consumption on acne vulgaris rather than a causative role in acne development. A different methodology may be needed to investigate a causative role.

We believe that this study can be considered as a starter for future focused research. However, as a randomized clinical trial, the results are strong enough to hypothesize an aggravating role for consumption of sunflower seeds in patients with acne vulgaris. It may also alert ethical considerations in design of future clinical trials. Therefore, the authors suggest future randomized clinical trials to be designed and conducted on an exposure elimination methodology rather than giving sunflower intake to those who do not usually consume it in their diet.

\subsection{Limitations and Strengths}

As like in majorities of randomized clinical trials, control of confounding was relied to be done through randomization for unmeasured-unknown confounders or through the analysis. The baseline severity of lesions was controlled through analysis in the present study, but factors such as hormonal status or other nutritional habits were assumed to be controlled through randomization, which is a reasonable approach in exploratory clinical trials. The small size and short term of investigation were other limitations of this study which re reasonable due to the early phase of the study. The main strengths in this study were the use of randomization for controlling possible confounding as well as the use of two internationally recommended clinical assessment tools.

\section{Authors' Contributions}

Alireza Mohebbipour: main investigator, design and carrying out the A-Z of the research and drafting; Homay- 
oun Sadeghi-Bazargani: statistical analysis, interpretation, drafting and review; Mona Mansouri: design, data collection, interpretation, drafting.

\section{Funding/Support}

This was an M.D. thesis study, supported by Ardabil Branch, Islamic Azad University, Ardabil, IR Iran.

\section{References}

1. Bowe WP, Joshi SS, Shalita AR. Diet and acne.J Am Acad Dermatol. 2010;63(1):124-41.

2. Davidovici BB, Wolf R. The role of diet in acne: facts and controversies. Clin Dermatol. 2010;28(1):12-6.

3. Ferdowsian HR, Levin S. Does diet really affect acne? Skin Therapy Lett. 2010;15(3):1-2.

4. Dweck AC. Skin treatment with plants of the Americas. Cosmet Tiol. 2011;112:47-64.

5. Kanlayavattanakul M, Lourith N. Therapeutic agents and herbs in topical application for acne treatment. Int $J$ Cosmet Sci. 2011;33(4):289-97.

6. Asghari-Jafarabadi M, Sadeghi-Bazargani H. Randomization: Techniques and Software-aided Implementation in Medical Studies. J Clin Res Govern. 2014;3(2).

7. Adalatkhah H, Pourfarzi F, Sadeghi-Bazargani H. Flutamide versus a cyproterone acetate-ethinyl estradiol combination in moderate acne: a pilot randomized clinical trial. Clin Cosmet Investig Dermatol. 2011;4:117-21.
8. Doshi A, Zaheer A, Stiller MJ. A comparison of current acne grading systems and proposal of a novel system. Int J Dermatol. 1997;36(6):416-8.

9. Tan J, Wolfe B, Weiss J, Stein-Gold L, Bikowski J, Del Rosso J, et al. Acne severity grading: determining essential clinical components and features using a Delphi consensus. J Am Acad Dermatol. 2012;67(2):187-93.

10. Fitzmaurice GM, Laird NM, Ware JH. Applied Longitudinal Analysis.: Wiley; 2004.

11. Spencer EH, Ferdowsian HR, Barnard ND. Diet and acne: a review of the evidence. Int J Dermatol. 2009;48(4):339-47.

12. Smith RN, Mann NJ, Braue A, Makelainen H, Varigos GA. A low-glycemic-load diet improves symptoms in acne vulgaris patients: a randomized controlled trial. Am J Clin Nutr. 2007;86(1):107-15.

13. Fulton JJ, Plewig G, Kligman AM. Effect of chocolate on acne vulgaris. JAMA. 1969;210(11):2071-4.

14. Smith RN, Braue A, Varigos GA, Mann NJ. The effect of a low glycemic load diet on acne vulgaris and the fatty acid composition of skin surface triglycerides. J Dermatol Sci. 2008;50(1):41-52.

15. Thiboutot DM, Strauss JS. Diet and acne revisited. Arch Dermatol. 2002;138(12):1591-2.

16. Cordain L, Lindeberg S, Hurtado M, Hill K, Eaton SB, Brand-Miller J. Acne vulgaris: a disease of Western civilization. Arch Dermatol. 2002;138(12):1584-90.

17. Abbott WG, Howard BV, Ruotolo G, Ravussin E. Energy expenditure in humans: effects of dietary fat and carbohydrate. Am J Physiol.1990;258(2 Pt 1):E347-51.

18. Tolkachev ON, Zhychenko AA. Biologically active substances of lax: medicinal and nutritional properties (a review). Pharm Chem J. 2000;34:360-7. 